\title{
Combining Ability Analysis for Yield Component and Biochemical Traits in Soybean [Glycine max (L.) Merrill]
}

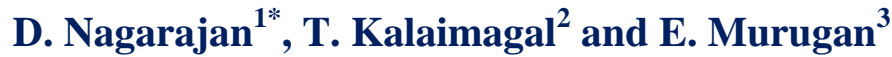 \\ ${ }^{1}$ Vanavarayar Institute of Agriculture, Manakkadavu, Pollachi, Tamil Nadu, India \\ ${ }^{2}$ Anbil Dharmalingam Agriculture College and Research Institute, TNAU, Trichy, \\ Tamil Nadu, India \\ ${ }^{3}$ Agricultural College and Research Institute, TNAU, Madurai, Tamil Nadu, India \\ *Corresponding author
}

\begin{tabular}{|c|c|}
\hline \multicolumn{2}{|r|}{ A B S T RA C T } \\
\hline & \multirow{7}{*}{$\begin{array}{l}\text { An investigation was taken up in soybean involving a set of five ovule parents (BRAGG, } \\
\text { RKS } 18 \text {, Co } 2 \text {, Co (Soy) } 3 \text { and JS 335) and five pollen parents (LPA 5-1, LPA 5-2, LPA 5- } \\
3 \text {, LPA } 13-1 \text { and LPA } 18 \text { ) crossed in a line } \times \text { tester mating fashion to study the general and } \\
\text { specific combining ability and the gene action determining the yield component and } \\
\text { biochemical traits. The lines are normal in phytic acid content. However, testers are low in } \\
\text { phytic acid content. Twenty five crosses were made between these lines and testers in } \\
\text { order to know the genetics of yield component and biochemical traits, during Kharif, 2013- } \\
\text { 14. Individual cross combinations along with their parents were studied for } 13 \text { characters } \\
\text { during Rabi } 2014 \text {. While comparing the performance of parents for per se and gca effects } \\
\text { for low phytic acid content and yield components Co (Soy) } 3 \text { and LPA 5-2 were } \\
\text { considered as superior parents for breeding programme. The crosses RKS } 18 \times \text { LPA 5-2, } \\
\text { Co (Soy) } 3 \times \text { LPA 5-2 and Co (Soy) } 3 \times \text { LPA 5-1, Co (Soy) } 3 \times \text { LPA } 13-1 \text { exhibited } \\
\text { superior per se performance and one of the parent possessed good general combining } \\
\text { ability for yield component and biochemical traits which indicates additive type of gene } \\
\text { action. }\end{array}$} \\
\hline Keywords & \\
\hline $\begin{array}{l}\text { Soybean, Combining } \\
\text { ability, Gene action, } \\
\text { Phytic acid content. }\end{array}$ & \\
\hline Article Info & \\
\hline & \\
\hline $\begin{array}{l}\text { Available Online: } \\
10 \text { November } 2017\end{array}$ & \\
\hline & \\
\hline
\end{tabular}

\section{Introduction}

Soybean is one of the most important legumes in many countries of the world which has been given recent predominance because of its broader utility and versatility for both human and animal nutrition. So, there is a need to increase the yield by selection of parents having good potentiality for yield and its related characters. The selection of suitable parents for an efficient breeding programme, the breeder has to know the genetic information especially about the nature of combining ability and the type of gene action governing the inheritance of economically important quantitative characters. Combining ability of genotypes gives essential information to select the genotypes as parents to produce better segregants and the knowledge of the type of gene action involved in the expression of yield and yield components is essential to choose an appropriate breeding strategy to isolate desirable segregants in the later generations. Therefore, the present investigation was taken up to study the combining ability parents for yield component and biochemical traits in soybean. 


\section{Materials and Methods}

Five ovule parents viz., BRAGG, RKS 18, Co 2, Co (Soy) 3 and JS 335 and five pollen parents, viz., LPA 5-1, LPA 5-2, LPA 5-3, LPA 13-1 and LPA 18 were raised in two staggered sowing at 3 days interval in order to achieve programmed pollination in the crossing block at the Department of Pulses, Tamil Nadu Agricultural University, Coimbatore during Rabi, 2013-14. The lines are normal in phytic acid content and testers are low in phytic acid content.

Female parents were raised in five rows of four meter length with a spacing of $60 \times 30 \mathrm{~cm}$ in order to ensure good growth and profuse flowering and fruiting.

Males were raised in two rows of four meter length by adopting a spacing of $30 \times 15 \mathrm{~cm}$. Crosses were made between these lines and testers in order to develop low phytic acid genotypes with high yield.

The parents were crossed in line $\times$ tester mating fashion to synthesize $25 \mathrm{~F}_{1}$ s. Crossed pods were collected and forwarded for $F_{1}$ evaluation.

Five plants were randomly selected in $\mathrm{F}_{1} \mathrm{~S}$ and their parents in each replication and observations were recorded for days to 50 per cent flowering, days to maturity, plant height $(\mathrm{cm})$, number of branches per plant, number of clusters per plant, number of pods per plant, number of seeds per pod, 100-seed weight $(\mathrm{g})$, seed yield per plant $(\mathrm{g})$, harvest index $(\%)$, phytic acid content $(\mathrm{mg} / \mathrm{g})$, Protein content (\%) and Oil content (\%). The mean values of five plants were utilized for estimation of general combining ability of parents and specific combining ability of hybrids for grain yield and its component traits using the Line $\mathrm{x}$ Tester analysis as suggested by Kempthorne (1957).

\section{Results and Discussion}

Analysis of variance indicated the presence of significant differences among genotypes for all the characters studied (Table 1). Significant variances were observed among hybrids and parents for all the characters. Higher magnitude of variance in case of hybrids as compared to parents had been observed for most of the characters indicating the presence of positive heterosis. However, negative heterosis is also desirable and observed for some characters viz., days to 50 per cent flowering, days to maturity and phytic acid content.

Analysis of variance for combining ability analysis (Table 2) indicated the presence of significant differences among the lines for all the characters studied except for days to maturity whereas significant differences were observed among the testers for all the characters except for days to 50 per cent flowering. While, significant differences were observed among the line $x$ tester interaction for all the characters except for days to 50 per cent flowering and days to maturity. The significant variance of line $x$ tester interaction indicated the preponderance of specific combining ability. The results showed the presence of considerable variability among the hybrids rather than among the lines and testers. Hence it is possible to select superior hybrids with high yield.

The magnitude of specific combining ability variances was much greater than those of general combining ability variances for all the characters studied except days to 50 per cent flowering indicating the preponderance of non-additive gene action in the expression of these traits (Table 3). Hence improvement of these yield related characters could be accomplished by selection at later filial generations. Similar results were observed by Mahesh et al., (2014). 


\section{Choice of parents}

The per se performance was considered as the first important criterion for evaluation. The per se performances of parents for yield components and biochemical traits were compared with general mean (Table 4). Based on per se, the line parents RKS 18 and Co (Soy) 3 recorded significantly superior mean for plant height, number of clusters per plant, number of pods per plant, seed yield per plant, harvest index and oil content.

Among the tester parent, LPA 5-1 recorded superior mean for plant height, number of clusters per plant, number of pods per plant, hundred seed weight, seed yield per plant and oil content and the parent LPA 5-2 exhibited the similar performance for number of clusters per plant, number of pods per plant, seed yield per plant, protein content and oil content. Hence the parents RKS 18, Co (Soy) 3, LPA 5-1, LPA 5-2 were considered as more superior than other parents for yield components and biochemical traits and were considered as desirable for recombination breeding.

The second criterion for selection is the general combining ability ( $\mathrm{gca}$ ) effects of the parents which is a factor that predicts the average performance of a line in a number of hybrid combinations (Table 5). Combining ability always decides the breeding value or genetic worth of the parent. The estimates of gca effect showed that among the lines, RKS 18 was found to be superior as it showed significant and positive gca effect plant height, number of branches per plant, number of clusters per plant, number of pods per plant, hundred seed weight, seed yield per plant, harvest index and oil content. The line parent Co (Soy) 3 was a good general combiner for plant height, number of branches per plant, number of clusters per plant, number of pods per plant, number of seeds per pod, hundred seed weight, seed yield per plant, harvest index, protein content and oil content, however, it recorded significant negative gca effect for phytic acid content. Among the tester parents, LPA 5-1 recorded significant positive $g c a$ effect for plant height, number of clusters per plant, number of pods per plant, hundred seed weight, seed yield per plant, and oil content while LPA 5-2 for number of branches per plant, number of clusters per plant, number of pods per plant, number of seeds per pod, hundred seed weight, seed yield per plant, harvest index, protein content and oil content, however, it recorded significant negative gca effect for phytic acid content.

Selection of parents based on per se performance and gca effects is of great importance in breeding programmes, because it provides useful information on the choice of parents in terms of expected performance of hybrids and progenies (Dhillon, 1975). While comparing the performance of parents for per se and gca effects for low phytic acid content and yield components Co (Soy) 3 and LPA 52 were considered as superior parents for breeding programme.

\section{Choice of crosses}

The specific combining ability ( $s c a$ ) effect alone may not be the appropriate choice for exploitation of heterosis because the hybrid with low mean value may also possess high sca effect. Hence, the cross combinations were identified based on two criteria viz., per se performance and the gene action involved in the crosses for further exploitation.

Based on per se performance for various traits, the crosses viz., RKS $18 \times$ LPA 5-2 Co (Soy) $3 \times$ LPA 5-2, Co (Soy) $3 \times$ LPA 5-1 and Co (Soy) $3 \times$ LPA 13-1 were considered as desirable crosses for yield along with low phytic acid content (Table 6). 
Table.1 Analysis of variance for parents and hybrids for yield and yield related traits in soybean

\begin{tabular}{|c|c|c|c|c|c|c|c|c|c|c|c|c|c|c|}
\hline Source & Df & $\begin{array}{c}\text { Days to } \\
50 \text { per } \\
\text { cent } \\
\text { flowering }\end{array}$ & $\begin{array}{c}\text { Days to } \\
\text { maturity }\end{array}$ & $\begin{array}{c}\text { Plant } \\
\text { height }\end{array}$ & $\begin{array}{c}\begin{array}{c}\text { Number } \\
\text { of } \\
\text { branches } \\
\text { per plant }\end{array} \\
\end{array}$ & $\begin{array}{c}\text { Number } \\
\text { of clusters } \\
\text { per plant }\end{array}$ & $\begin{array}{c}\text { Number } \\
\text { of pods } \\
\text { per plant }\end{array}$ & $\begin{array}{l}\text { Number } \\
\text { of seeds } \\
\text { per pod }\end{array}$ & $\begin{array}{c}\text { Hundred } \\
\text { seed } \\
\text { weight }\end{array}$ & $\begin{array}{c}\text { Seed } \\
\text { yield per } \\
\text { plant }\end{array}$ & $\begin{array}{c}\text { Harvest } \\
\text { index }\end{array}$ & $\begin{array}{c}\text { Phytic } \\
\text { acid } \\
\text { content }\end{array}$ & $\begin{array}{l}\text { Protein } \\
\text { content }\end{array}$ & $\begin{array}{c}\text { Oil } \\
\text { content }\end{array}$ \\
\hline Hybrids & 24 & $7.75^{*}$ & $13.98^{* *}$ & $99.21 * *$ & $1.51 * *$ & $58.92 * *$ & $1248.45^{* *}$ & 0.06 ** & $2.84 * *$ & $139.78 * *$ & $54.21 * *$ & $1.85 * *$ & $4.72 * *$ & $8.44 * *$ \\
\hline Parents & 9 & $8.89 *$ & $24.56^{* *}$ & $263.75 * *$ & $1.29 * *$ & $70.55^{* *}$ & $1663.32^{* *}$ & $0.11^{* *}$ & $1.36 * *$ & $55.47 * *$ & $31.26^{* *}$ & $6.13 * *$ & $4.47 * *$ & $2.41 * *$ \\
\hline Hybrids vs Parents & 1 & $33.44 * *$ & $67.27 * *$ & 8.38 & $6.04 * *$ & $39.18 * *$ & $192.62 * *$ & 0.00 & 0.38 & $15.05^{* *}$ & $168.54 * *$ & $2.18^{* *}$ & 0.65 & $2.13^{* *}$ \\
\hline Error & 34 & 4.09 & 3.77 & 4.37 & 0.09 & 1.35 & 8.32 & 0.01 & 0.15 & 2.06 & 3.91 & 0.04 & 0.24 & 0.12 \\
\hline
\end{tabular}

*, ** Significant at $5 \%$ and $1 \%$ level of probability, respectively.

Table.2 Analysis of variance for combining ability analysis for yield and yield related traits in soybean

\begin{tabular}{|c|c|c|c|c|c|c|c|c|c|c|c|c|c|c|}
\hline Source & df & $\begin{array}{c}\text { Days to } \\
50 \text { per } \\
\text { cent } \\
\text { flowering }\end{array}$ & $\begin{array}{l}\text { Days to } \\
\text { maturity }\end{array}$ & $\begin{array}{l}\text { Plant } \\
\text { height }\end{array}$ & $\begin{array}{c}\text { Number } \\
\text { of } \\
\text { branches } \\
\text { per plant }\end{array}$ & $\begin{array}{l}\text { Number } \\
\text { of clusters } \\
\text { per plant }\end{array}$ & $\begin{array}{c}\text { Number } \\
\text { of pods } \\
\text { per plant }\end{array}$ & $\begin{array}{l}\text { Number } \\
\text { of seeds } \\
\text { per pod }\end{array}$ & $\begin{array}{c}\text { Hundred } \\
\text { seed } \\
\text { weight }\end{array}$ & $\begin{array}{c}\text { Seed yield } \\
\text { per plant }\end{array}$ & $\begin{array}{l}\text { Harvest } \\
\text { index }\end{array}$ & $\begin{array}{l}\text { Phytic } \\
\text { acid } \\
\text { content }\end{array}$ & $\begin{array}{l}\text { Protein } \\
\text { content }\end{array}$ & $\begin{array}{c}\text { Oil } \\
\text { content }\end{array}$ \\
\hline Lines & 4 & $26.72 * *$ & 12.77 & $406.15^{* *}$ & $4.53 * *$ & $241.18 * *$ & $4659.70 * *$ & $0.04 *$ & 10.18 ** & $427.32 * *$ & $154.46^{* * *}$ & $3.55^{* *}$ & $4.12 * *$ & $33.90 * *$ \\
\hline Testers & 4 & 10.22 & $41.57 * *$ & $117.95^{* *}$ & $2.99 * *$ & $68.50 * *$ & $1835.44 * *$ & $0.06^{* *}$ & $1.37 * *$ & $207.39 * *$ & $130.08 * *$ & $2.75^{* *}$ & $9.36^{* *}$ & $6.61 * *$ \\
\hline $\mathrm{L} \times \mathrm{T}$ & 16 & 2.38 & 7.38 & $17.79 * *$ & $0.38 * *$ & $10.97 * *$ & $248.89^{* *}$ & $0.06^{* *}$ & $1.37 * *$ & $50.99 * *$ & $10.19 * *$ & $1.19 * *$ & $3.72 * *$ & $2.54 * *$ \\
\hline Error & 24 & 5.42 & 4.75 & 5.42 & 0.11 & 1.04 & 9.42 & 0.01 & 0.14 & 2.18 & 4.19 & 0.04 & 0.27 & 0.11 \\
\hline
\end{tabular}

*, ** Significant at $5 \%$ and $1 \%$ level of probability, respectively.

Table.3 Magnitude of combining ability variance for yield and yield related traits in soybean

\begin{tabular}{lccc}
\hline \multicolumn{1}{c}{ Characters } & GCA & SCA & GCA/SCA \\
\hline Days to 50 per cent flowering & 0.20 & -1.52 & -0.13 \\
Days to maturity & 0.25 & 1.32 & 0.19 \\
Plant height & 3.05 & 6.19 & 0.49 \\
Number of branches per plant & 0.04 & 0.13 & 0.31 \\
Number of clusters per plant & 1.80 & 4.96 & 0.36 \\
Number of pods per plant & 37.48 & 119.73 & 0.31 \\
Number of seeds per pod & -0.01 & 0.02 & -0.41 \\
Hundred seed weight & 0.06 & 0.61 & 0.09 \\
Seed yield per plant & 3.33 & 24.40 & 0.14 \\
Harvest index & 1.65 & 3.00 & 0.55 \\
Phytic acid content & 0.02 & 0.58 & 0.04 \\
Protein content & 0.04 & 1.72 & 0.02 \\
Oil content & 0.22 & 1.21 & 0.18 \\
\hline
\end{tabular}


Table.4 Per se performance of parents for yield and yield related traits in soybean

\begin{tabular}{|c|c|c|c|c|c|c|c|c|c|c|c|c|c|}
\hline Parents & $\begin{array}{c}\text { Days to } 50 \\
\text { per cent } \\
\text { flowering }\end{array}$ & $\begin{array}{c}\text { Days to } \\
\text { maturity }\end{array}$ & $\begin{array}{c}\text { Plant } \\
\text { height } \\
(\mathbf{c m})\end{array}$ & $\begin{array}{l}\text { Number of } \\
\text { branches } \\
\text { per plant }\end{array}$ & $\begin{array}{c}\text { Number of } \\
\text { clusters } \\
\text { per plant }\end{array}$ & $\begin{array}{c}\text { Number of } \\
\text { pods per } \\
\text { plant }\end{array}$ & $\begin{array}{c}\text { Number } \\
\text { of seeds } \\
\text { per pod }\end{array}$ & $\begin{array}{l}\text { Hundred } \\
\text { seed } \\
\text { weight (g) }\end{array}$ & $\begin{array}{c}\text { Seed yield } \\
\text { per plant } \\
\text { (g) }\end{array}$ & $\begin{array}{c}\text { Harvest } \\
\text { index }\end{array}$ & $\begin{array}{l}\text { Phytic } \\
\text { acid } \\
\text { content } \\
(\mathrm{mg} / \mathrm{g})\end{array}$ & $\begin{array}{c}\text { Protein } \\
\text { content } \\
(\%)\end{array}$ & $\begin{array}{c}\text { Oil } \\
\text { content } \\
(\%)\end{array}$ \\
\hline \multicolumn{14}{|l|}{ Lines } \\
\hline BRAGG & $33.00^{\mathrm{a}}$ & $85.50^{\mathrm{a}}$ & 26.10 & 5.20 & 16.10 & 67.40 & $2.30^{\mathrm{a}}$ & 9.85 & 14.92 & $0.50^{\mathrm{a}}$ & 5.11 & 35.94 & $17.34^{\mathrm{a}}$ \\
\hline RKS 18 & $36.00^{\mathrm{a}}$ & 89.00 & $50.00^{* * *}$ & $6.20^{\mathrm{a}}$ & $27.60^{* *}$ & $119.70 * *$ & $2.00^{\mathrm{a}}$ & $11.30^{\mathrm{a}}$ & $26.05^{* * *}$ & $0.57 *$ & 6.23 & 33.91 & 18.27 ** \\
\hline $\operatorname{Co} 2$ & $33.50^{\mathrm{a}}$ & 88.00 & 23.60 & 5.30 & 13.60 & 59.60 & $2.00^{\mathrm{a}}$ & $10.33^{\mathrm{a}}$ & 12.53 & $0.49^{\mathrm{a}}$ & 5.10 & $37.00^{\mathrm{a}}$ & $16.96^{\mathrm{a}}$ \\
\hline Co (Soy) 3 & $36.00^{\mathrm{a}}$ & $86.00^{\mathrm{a}}$ & $49.60^{* * *}$ & $6.10^{\mathrm{a}}$ & $26.00^{* * *}$ & $117.30 * *$ & $2.00^{\mathrm{a}}$ & $11.03^{\mathrm{a}}$ & $25.27 * *$ & $0.55^{\mathrm{a}}$ & 6.03 & $36.14^{\mathrm{a}}$ & $18.56^{* * *}$ \\
\hline JS 335 & $30.50^{\mathrm{a}}$ & $79.50^{\mathrm{a}}$ & 24.90 & 4.70 & 12.40 & 50.50 & $2.50 * *$ & $11.07^{\mathrm{a}}$ & 13.84 & 0.46 & 5.29 & $38.44 *$ & 16.27 \\
\hline \multicolumn{14}{|l|}{ Testers } \\
\hline LPA 5-1 & $35.50^{\mathrm{a}}$ & $82.50^{\mathrm{a}}$ & 51.80 *** & $6.30^{\mathrm{a}}$ & 27.20 ** & $121.30 * * *$ & $2.10^{\mathrm{a}}$ & $11.99 *$ & $24.48 * *$ & $0.54^{\mathrm{a}}$ & $2.02 * *$ & $36.90^{\mathrm{a}}$ & $19.05^{* *}$ \\
\hline LPA 5-2 & $36.00^{\mathrm{a}}$ & $84.00^{\mathrm{a}}$ & $34.20^{\mathrm{a}}$ & $5.90^{\mathrm{a}}$ & $25.60^{* *}$ & $104.00^{* * *}$ & $2.00^{\mathrm{a}}$ & $10.96^{\mathrm{a}}$ & $22.73^{*}$ & $0.52^{\mathrm{a}}$ & $1.90 * *$ & $38.77 * *$ & $18.19 * *$ \\
\hline LPA 5-3 & $36.50^{\mathrm{a}}$ & $83.00^{\mathrm{a}}$ & $37.40^{\mathrm{a}}$ & $5.70^{\mathrm{a}}$ & 17.60 & 75.90 & $2.00^{\mathrm{a}}$ & $10.73^{a}$ & 15.67 & 0.46 & $2.73 * *$ & $38.35^{*}$ & $16.98^{\mathrm{a}}$ \\
\hline LPA $13-1$ & $35.00^{\mathrm{a}}$ & $86.00^{\mathrm{a}}$ & $42.20^{* *}$ & $5.80^{\mathrm{a}}$ & $19.20^{\mathrm{a}}$ & $87.50^{\mathrm{a}}$ & $2.00^{\mathrm{a}}$ & $10.95^{\mathrm{a}}$ & $18.10^{\mathrm{a}}$ & 0.49 & $3.06 * *$ & $37.70^{\mathrm{a}}$ & $17.82^{\mathrm{a}}$ \\
\hline LPA 18 & $31.50^{\mathrm{a}}$ & $78.00 *$ & 24.20 & 3.70 & 15.50 & 48.60 & $2.60 * *$ & $12.82^{* *}$ & 14.53 & 0.46 & $2.11 * *$ & $38.12^{\mathrm{a}}$ & 15.49 \\
\hline General mean & 33.26 & 82.60 & 35.85 & 5.95 & 21.26 & 87.80 & 2.14 & 10.98 & 19.54 & 0.53 & 3.68 & 37.28 & 17.22 \\
\hline S.E. & 1.43 & 1.37 & 1.48 & 0.21 & 0.82 & 2.04 & 0.07 & 0.27 & 1.02 & 1.4 & 0.14 & 0.35 & 0.25 \\
\hline $\mathrm{CD}(\mathrm{P}=0.05)$ & 4.07 & 3.90 & 4.20 & 0.60 & 2.34 & 5.80 & 0.21 & 0.78 & 2.89 & 3.97 & 0.39 & 0.98 & 0.70 \\
\hline $\mathrm{CD}(\mathrm{P}=0.01)$ & 5.42 & 5.20 & 5.60 & 0.80 & 3.12 & 7.73 & 0.28 & 1.03 & 3.85 & 5.30 & 0.52 & 1.31 & 0.94 \\
\hline
\end{tabular}

${ }^{\mathrm{a}}$ and $* * *$ On par and significantly superior than the general mean at $5 \%, 1 \%$ level of probability, respectively.

Table.5 Estimates of general combining ability ( $\mathrm{gca}$ ) effects for yield and yield related traits in soybean

\begin{tabular}{|c|c|c|c|c|c|c|c|c|c|c|c|c|c|}
\hline Parents & $\begin{array}{c}\text { Days to } 50 \\
\text { per cent } \\
\text { flowering }\end{array}$ & $\begin{array}{c}\text { Days to } \\
\text { maturity }\end{array}$ & $\begin{array}{l}\text { Plant } \\
\text { height }\end{array}$ & $\begin{array}{l}\text { Number of } \\
\text { branches } \\
\text { per plant }\end{array}$ & $\begin{array}{c}\text { Number of } \\
\text { clusters per } \\
\text { plant }\end{array}$ & $\begin{array}{c}\text { Number } \\
\text { of pods } \\
\text { per plant }\end{array}$ & $\begin{array}{l}\text { Number of } \\
\text { seeds per } \\
\text { pod }\end{array}$ & $\begin{array}{c}\text { Hundred } \\
\text { seed } \\
\text { weight }\end{array}$ & $\begin{array}{c}\text { Seed } \\
\text { yield per } \\
\text { plant }\end{array}$ & $\begin{array}{l}\text { Harvest } \\
\text { index }\end{array}$ & $\begin{array}{l}\text { Phytic } \\
\text { acid } \\
\text { content }\end{array}$ & $\begin{array}{l}\text { Protein } \\
\text { content }\end{array}$ & $\begin{array}{c}\text { Oil } \\
\text { content }\end{array}$ \\
\hline \multicolumn{14}{|l|}{ Lines } \\
\hline BRAGG & 0.38 & 0.42 & $-6.36 * *$ & $-0.90 * *$ & $-3.42 * *$ & $-14.33 * *$ & -0.06 & $-1.68 * *$ & $-6.65 * *$ & $-4.77 * *$ & $0.82 * *$ & -0.15 & $-1.97 * *$ \\
\hline RKS 18 & 0.78 & 1.32 & $6.43 * *$ & $0.56^{* *}$ & 4.88 ** & $20.79 * *$ & 0.00 & $0.33 * *$ & $5.23 * *$ & $3.54 * *$ & $0.41 * *$ & 0.04 & $1.71 * *$ \\
\hline $\mathrm{Co} 2$ & 1.38 & 0.62 & $-3.41 * *$ & 0.08 & $-3.84 * *$ & $-15.21 * *$ & -0.06 & -0.09 & $-3.92 * *$ & 0.09 & $-0.35 * *$ & -0.32 & $-1.00 * *$ \\
\hline Co (Soy) 3 & 0.28 & -1.08 & $7.31 * *$ & $0.70 * *$ & $5.84 * *$ & $26.25 * *$ & $0.10 * *$ & $0.93 * *$ & $8.58 * *$ & $4.16 * *$ & $-0.65 * *$ & $1.06 * *$ & $2.21 * *$ \\
\hline JS 335 & $-2.82 * *$ & -1.28 & $-3.95 * *$ & $-0.44 * *$ & $-3.48 * *$ & $-17.49 * *$ & 0.00 & $0.51 * *$ & $-3.24 * *$ & $-3.03 * *$ & $-0.23 * *$ & $-0.63 * *$ & $-0.94 * *$ \\
\hline \multicolumn{14}{|l|}{ Testers } \\
\hline LPA 5-1 & -0.62 & 0.52 & $1.81 *$ & 0.06 & $1.62 * *$ & 6.79 ** & -0.04 & $0.36 * *$ & $1.68 * *$ & 0.57 & $0.32 * *$ & 0.28 & 0.11 \\
\hline LPA 5-2 & 0.18 & -0.88 & $-1.74 *$ & $0.60 * *$ & 2.96 ** & $14.01 * *$ & $0.12 * *$ & $0.43 * *$ & $6.20 * *$ & 4.84 ** & $-0.84 * *$ & $1.09 * *$ & $0.68 * *$ \\
\hline LPA 5-3 & $1.68 *$ & $2.02 * *$ & 1.23 & $0.24 *$ & 0.04 & 0.33 & -0.06 & -0.15 & -0.09 & -0.11 & -0.02 & $0.56 * *$ & $0.44 * *$ \\
\hline LPA 13-1 & -0.82 & $1.42 *$ & $3.75 * *$ & -0.02 & $-0.70 *$ & 1.03 & -0.06 & $-0.26 *$ & $-1.52 * *$ & 0.03 & $0.54 * *$ & $-0.60 * *$ & 0.15 \\
\hline LPA 18 & -0.42 & $-3.08 * *$ & $-5.03 * *$ & $-0.88 * *$ & $-3.94 * *$ & $-22.15 * *$ & 0.02 & $-0.38 * *$ & $-6.27 * *$ & $-5.32 * *$ & 0.00 & $-1.34 * *$ & $-1.39 * *$ \\
\hline S.E. (gca effects) & 0.74 & 0.69 & 0.74 & 0.10 & 0.32 & 0.97 & 0.04 & 0.12 & 0.47 & 0.65 & 0.06 & 0.17 & 0.10 \\
\hline
\end{tabular}

$*$, ** Significant at $5 \%$ and $1 \%$ level of probability, respectively. 
Table.6 Per se performance of hybrids for yield and yield related traits in soybean

\begin{tabular}{|c|c|c|c|c|c|c|c|c|c|c|c|c|c|}
\hline Hybrids & $\begin{array}{c}\text { Days to } 50 \\
\text { per cent } \\
\text { flowering }\end{array}$ & $\begin{array}{c}\text { Days to } \\
\text { maturity }\end{array}$ & $\begin{array}{c}\text { Plant } \\
\text { height } \\
(\mathrm{cm})\end{array}$ & $\begin{array}{c}\begin{array}{c}\text { Number } \\
\text { of } \\
\text { branches }\end{array} \\
\text { per plant }\end{array}$ & $\begin{array}{c}\text { Number } \\
\text { of } \\
\text { clusters } \\
\text { per plant }\end{array}$ & $\begin{array}{c}\text { Number } \\
\text { of pods } \\
\text { per } \\
\text { plant }\end{array}$ & $\begin{array}{c}\text { Number } \\
\text { of seeds } \\
\text { per pod }\end{array}$ & $\begin{array}{l}\text { Hundred } \\
\text { seed } \\
\text { weight } \\
\text { (g) }\end{array}$ & $\begin{array}{c}\text { Seed } \\
\text { yield per } \\
\text { plant (g) }\end{array}$ & $\begin{array}{c}\text { Harvest } \\
\text { index }\end{array}$ & $\begin{array}{l}\text { Phytic } \\
\text { acid } \\
\text { content } \\
\text { (mg/g) }\end{array}$ & $\begin{array}{c}\text { Protein } \\
\text { content } \\
(\%)\end{array}$ & $\begin{array}{c}\text { Oil } \\
\text { content } \\
(\%)\end{array}$ \\
\hline BRAGG X LPA 5-1 & $33.50^{\mathrm{a}}$ & $80.50^{\mathrm{a}}$ & $33.00^{\mathrm{a}}$ & 5.30 & 18.70 & 72.50 & $2.10^{\mathrm{a}}$ & $11.36^{\mathrm{a}}$ & 16.38 & 0.49 & 4.22 & 36.03 & 16.08 \\
\hline BRAGG X LPA 5-2 & $34.00^{\mathrm{a}}$ & $83.00^{\mathrm{a}}$ & 24.85 & $5.50^{\mathrm{a}}$ & 18.60 & 76.50 & $2.00^{\mathrm{a}}$ & 8.64 & 12.59 & $0.54^{\mathrm{a}}$ & $3.74^{\mathrm{a}}$ & 36.23 & 15.01 \\
\hline BRAGG X LPA 5-3 & $36.50^{\mathrm{a}}$ & $86.00^{\mathrm{a}}$ & 28.10 & 5.30 & $19.50^{\mathrm{a}}$ & 76.30 & $2.00^{\mathrm{a}}$ & 9.13 & 12.68 & 0.48 & 4.86 & $37.39^{\mathrm{a}}$ & 14.52 \\
\hline BRAGG X LPA 13-1 & $31.00^{\mathrm{a}}$ & $85.50^{\mathrm{a}}$ & $34.60^{\mathrm{a}}$ & 5.20 & 17.90 & 80.50 & $2.00^{\mathrm{a}}$ & 9.12 & 13.21 & $0.49^{\mathrm{a}}$ & 5.04 & $37.83^{\mathrm{a}}$ & 15.45 \\
\hline BRAGG X LPA 18 & $31.00^{\mathrm{a}}$ & $77.00^{* *}$ & 25.80 & 4.90 & 16.90 & 66.80 & $2.30^{\mathrm{a}}$ & 8.05 & 11.07 & 0.45 & $4.05^{\mathrm{a}}$ & $38.47 *$ & 14.63 \\
\hline RKS 18 X LPA 5-1 & $33.00^{\mathrm{a}}$ & $84.50^{\mathrm{a}}$ & $43.30 * *$ & $6.70 *$ & $29.60 * *$ & $126.80^{* *}$ & $2.00^{\mathrm{a}}$ & $10.98^{\mathrm{a}}$ & $26.36 * *$ & $0.58 *$ & 5.01 & $38.73 * *$ & $18.32 * *$ \\
\hline RKS 18 X LPA 5-2 & $32.50^{\mathrm{a}}$ & $79.00^{\mathrm{a}}$ & $43.20 * *$ & $7.60 * *$ & $33.30 * *$ & $138.20 * *$ & $2.50 * *$ & $12.87 * *$ & $39.59 * *$ & $0.64 * *$ & $2.26 * *$ & $39.70 * *$ & $20.31 * *$ \\
\hline RKS 18 X LPA 5-3 & $34.50^{\mathrm{a}}$ & $85.50^{\mathrm{a}}$ & $43.40 * *$ & $6.80 * *$ & $26.60 * *$ & $102.50 * *$ & $2.10^{\mathrm{a}}$ & $11.40^{\mathrm{a}}$ & $23.95 * *$ & $0.57 *$ & $2.72 * *$ & $37.75^{\mathrm{a}}$ & $19.73 * *$ \\
\hline RKS 18 X LPA $13-1$ & $33.50^{\mathrm{a}}$ & 87.00 & $47.90 * *$ & $6.30^{\mathrm{a}}$ & $24.50 * *$ & $105.20 * *$ & $2.00^{\mathrm{a}}$ & $10.61^{\mathrm{a}}$ & $20.85^{\mathrm{a}}$ & $0.56^{\mathrm{a}}$ & 5.66 & 35.68 & $18.76^{* * *}$ \\
\hline RKS 18 X LPA 18 & $34.50^{\mathrm{a}}$ & $80.50^{\mathrm{a}}$ & 32.50 & $6.10^{\mathrm{a}}$ & $19.10^{\mathrm{a}}$ & 75.50 & $2.10^{\mathrm{a}}$ & $10.50^{\mathrm{a}}$ & 14.58 & $0.51^{\mathrm{a}}$ & 4.19 & 35.03 & $16.97^{\mathrm{a}}$ \\
\hline Co 2 X LPA 5-1 & $33.50^{\mathrm{a}}$ & $84.50^{\mathrm{a}}$ & $37.60^{\mathrm{a}}$ & $6.40^{\mathrm{a}}$ & 18.50 & 76.90 & $2.00^{\mathrm{a}}$ & $10.66^{\mathrm{a}}$ & 15.69 & $0.53^{\mathrm{a}}$ & $4.01^{\mathrm{a}}$ & $38.45^{*}$ & $16.58^{\mathrm{a}}$ \\
\hline Co 2 X LPA 5-2 & $33.50^{\mathrm{a}}$ & $83.50^{\mathrm{a}}$ & 25.70 & 6.50 & 17.30 & 71.80 & $2.00^{\mathrm{a}}$ & $10.28^{\mathrm{a}}$ & 14.61 & $0.55^{\mathrm{a}}$ & $3.16^{* *}$ & $37.17^{\mathrm{a}}$ & 15.09 \\
\hline Co 2 X LPA 5-3 & $36.00^{\mathrm{a}}$ & $84.50^{\mathrm{a}}$ & $35.50^{\mathrm{a}}$ & $6.80 * *$ & $20.70^{\mathrm{a}}$ & $89.70^{\mathrm{a}}$ & $2.30^{\mathrm{a}}$ & $11.35^{\mathrm{a}}$ & $23.57 * *$ & $0.58 * *$ & $2.57 * *$ & $38.73 * *$ & $18.44 * *$ \\
\hline Co 2 X LPA $13-1$ & $33.50^{\mathrm{a}}$ & $81.50^{\mathrm{a}}$ & $35.30^{\mathrm{a}}$ & $6.50^{\mathrm{a}}$ & 17.40 & 73.10 & $2.00^{\mathrm{a}}$ & $10.60^{\mathrm{a}}$ & 13.64 & $0.55^{\mathrm{a}}$ & $3.56^{\mathrm{a}}$ & 35.49 & 15.68 \\
\hline Co 2 X LPA 18 & $34.50^{\mathrm{a}}$ & $79.00^{\mathrm{a}}$ & 27.00 & 4.90 & 15.60 & 56.70 & $2.10^{\mathrm{a}}$ & $11.33^{\mathrm{a}}$ & 12.08 & 0.48 & $2.79 * *$ & 35.28 & 14.74 \\
\hline
\end{tabular}

Table.6 Continued...

\begin{tabular}{|c|c|c|c|c|c|c|c|c|c|c|c|c|c|}
\hline Hybrids & $\begin{array}{c}\text { Days to } 50 \\
\text { per cent } \\
\text { flowering }\end{array}$ & $\begin{array}{c}\text { Days to } \\
\text { maturity }\end{array}$ & $\begin{array}{c}\text { Plant } \\
\text { height } \\
(\mathrm{cm})\end{array}$ & $\begin{array}{c}\text { Number } \\
\text { of } \\
\text { branches } \\
\text { per plant }\end{array}$ & $\begin{array}{c}\text { Number } \\
\text { of } \\
\text { clusters } \\
\text { per plant }\end{array}$ & $\begin{array}{c}\text { Number } \\
\text { of pods } \\
\text { per } \\
\text { plant }\end{array}$ & $\begin{array}{l}\text { Number } \\
\text { of seeds } \\
\text { per pod }\end{array}$ & $\begin{array}{l}\text { Hundred } \\
\text { seed } \\
\text { weight } \\
\text { (g) }\end{array}$ & $\begin{array}{c}\text { Seed } \\
\text { yield per } \\
\text { plant (g) }\end{array}$ & $\begin{array}{c}\text { Harvest } \\
\text { index }\end{array}$ & $\begin{array}{c}\text { Phytic } \\
\text { acid } \\
\text { content } \\
(\mathrm{mg} / \mathrm{g})\end{array}$ & $\begin{array}{c}\text { Protein } \\
\text { content } \\
(\%)\end{array}$ & $\begin{array}{c}\text { Oil } \\
\text { content } \\
(\%)\end{array}$ \\
\hline Co (Soy) 3 X LPA 5-1 & $32.50^{\mathrm{a}}$ & $81.00^{\mathrm{a}}$ & 43.60 ** & $7.20 * *$ & $29.70 * *$ & $131.30 * *$ & $2.40 *$ & $12.01 * *$ & $33.73 * *$ & $0.62 * *$ & $2.75 * *$ & $37.64^{\mathrm{a}}$ & $20.27 * *$ \\
\hline Co (Soy) 3 X LPA 5-2 & $33.50^{\mathrm{a}}$ & $79.50^{\mathrm{a}}$ & $44.30 * *$ & $7.90 * *$ & $32.50 * *$ & $137.60 * *$ & $2.50 * *$ & $12.84 * *$ & $39.57 * *$ & $0.64 * *$ & $2.00 * *$ & $40.18 * *$ & $20.89 * *$ \\
\hline Co (Soy) 3 X LPA 5-3 & $34.50^{\mathrm{a}}$ & $82.00^{\mathrm{a}}$ & $45.00 * *$ & $7.20 * *$ & $25.20 * *$ & $106.70 * *$ & $2.00^{\mathrm{a}}$ & $11.05^{\mathrm{a}}$ & $23.52 * *$ & $0.55^{\mathrm{a}}$ & $3.56^{\mathrm{a}}$ & $38.93 * *$ & $19.03 * *$ \\
\hline Co (Soy) 3 X LPA 13-1 & $32.00^{\mathrm{a}}$ & $81.50^{\mathrm{a}}$ & $44.20 * *$ & $6.90 * *$ & $28.20 * *$ & $117.70 * *$ & $2.30^{\mathrm{a}}$ & $12.03 * *$ & $28.70^{* *}$ & $0.58 *$ & $2.42 * *$ & $38.98 * *$ & $19.93 * *$ \\
\hline Co (Soy) 3 X LPA 18 & $33.00^{\mathrm{a}}$ & $80.50^{\mathrm{a}}$ & $37.60^{\mathrm{a}}$ & 5.00 & $22.30^{\mathrm{a}}$ & $82.20^{\mathrm{a}}$ & $2.00^{\mathrm{a}}$ & $11.43^{\mathrm{a}}$ & 16.54 & $0.50^{\mathrm{a}}$ & $3.86^{\mathrm{a}}$ & 36.27 & 16.44 \\
\hline JS 335 X LPA 5-1 & $28.50 *$ & $82.00^{\mathrm{a}}$ & 29.70 & $5.40^{\mathrm{a}}$ & $20.30^{\mathrm{a}}$ & 70.70 & $2.00^{\mathrm{a}}$ & $11.46^{\mathrm{a}}$ & 15.44 & $0.50^{\mathrm{a}}$ & $3.43^{\mathrm{a}}$ & $37.28^{\mathrm{a}}$ & 14.85 \\
\hline JS 335 X LPA 5-2 & $31.50^{\mathrm{a}}$ & $80.50^{\mathrm{a}}$ & 31.40 & $6.20^{\mathrm{a}}$ & $21.80^{\mathrm{a}}$ & $90.20^{\mathrm{a}}$ & $2.30^{\mathrm{a}}$ & $12.23 * *$ & $23.83 * *$ & $0.55^{\mathrm{a}}$ & $2.45 * *$ & $38.89 * *$ & $17.65^{\mathrm{a}}$ \\
\hline JS 335 X LPA 5-3 & $31.00^{\mathrm{a}}$ & $82.00^{\mathrm{a}}$ & $32.30^{\mathrm{a}}$ & $5.80^{\mathrm{a}}$ & 16.90 & 70.70 & $2.00^{\mathrm{a}}$ & $11.01^{\mathrm{a}}$ & 14.98 & $0.49^{\mathrm{a}}$ & $4.01^{\mathrm{a}}$ & $36.69^{a}$ & 16.03 \\
\hline JS 335 X LPA 13-1 & $30.00^{\mathrm{a}}$ & $81.50^{\mathrm{a}}$ & $34.90^{\mathrm{a}}$ & $5.70^{\mathrm{a}}$ & 17.20 & 72.90 & $2.10^{\mathrm{a}}$ & $11.04^{\mathrm{a}}$ & 15.19 & $0.51^{\mathrm{a}}$ & $3.83^{\mathrm{a}}$ & 35.73 & 16.48 \\
\hline JS 335 X LPA 18 & $29.00 *$ & $77.50^{*}$ & 30.10 & $5.40^{\mathrm{a}}$ & 15.10 & 52.30 & $2.30^{\mathrm{a}}$ & $11.49^{\mathrm{a}}$ & 13.55 & 0.48 & $2.96 * *$ & 34.95 & 15.79 \\
\hline General mean & 33.26 & 82.60 & 35.85 & 5.95 & 21.26 & 87.80 & 2.14 & 10.98 & 19.54 & 52.73 & 3.68 & 37.28 & 17.22 \\
\hline S.E. & 1.43 & 1.37 & 1.48 & 0.21 & 0.82 & 2.04 & 0.07 & 0.27 & 1.02 & 1.4 & 0.14 & 0.35 & 0.25 \\
\hline $\mathrm{CD}(\mathrm{P}=0.05)$ & 4.07 & 3.90 & 4.20 & 0.60 & 2.34 & 5.80 & 0.21 & 0.78 & 2.89 & 3.97 & 0.39 & 0.98 & 0.70 \\
\hline $\mathrm{CD}(\mathrm{P}=0.01)$ & 5.42 & 5.20 & 5.60 & 0.80 & 3.12 & 7.73 & 0.28 & 1.03 & 3.85 & 5.30 & 0.52 & 1.31 & 0.94 \\
\hline
\end{tabular}

${ }^{\mathrm{a}}$ and $* * *$ On par and significantly superior than the general mean at $5 \%, 1 \%$ level of probability, respectively. 
Table.7 Estimates of specific combining ability (sca) effects for yield and yield related traits in soybean

\begin{tabular}{|c|c|c|c|c|c|c|c|c|c|c|c|c|c|}
\hline Hybrids & $\begin{array}{c}\text { Days to } 50 \\
\text { per cent } \\
\text { flowering }\end{array}$ & $\begin{array}{c}\text { Days to } \\
\text { maturity }\end{array}$ & $\begin{array}{l}\text { Plant } \\
\text { height }\end{array}$ & $\begin{array}{c}\begin{array}{c}\text { Number } \\
\text { of } \\
\text { branches }\end{array} \\
\text { per plant }\end{array}$ & $\begin{array}{l}\text { Number } \\
\text { of clusters } \\
\text { per plant }\end{array}$ & $\begin{array}{c}\text { Number } \\
\text { of pods } \\
\text { per } \\
\text { plant }\end{array}$ & $\begin{array}{c}\text { Number } \\
\text { of seeds } \\
\text { per pod }\end{array}$ & $\begin{array}{c}\text { Hundred } \\
\text { seed } \\
\text { weight }\end{array}$ & $\begin{array}{c}\text { Seed } \\
\text { yield } \\
\text { per } \\
\text { plant }\end{array}$ & $\begin{array}{c}\text { Harvest } \\
\text { index }\end{array}$ & $\begin{array}{l}\text { Phytic } \\
\text { acid } \\
\text { content }\end{array}$ & $\begin{array}{l}\text { Protein } \\
\text { content }\end{array}$ & $\begin{array}{c}\text { Oil } \\
\text { content }\end{array}$ \\
\hline BRAGG X LPA 5-1 & 0.92 & -2.42 & 1.92 & 0.00 & -1.24 & $-8.81 * *$ & 0.06 & $1.74 * *$ & 1.51 & -0.82 & $-0.48 * *$ & $-1.44 * *$ & $0.83 * *$ \\
\hline BRAGG X LPA 5-2 & 0.62 & 1.48 & -2.68 & -0.34 & $-2.68 * *$ & $-12.03 * *$ & $-0.20 *$ & $-1.05 * *$ & $-6.80 * *$ & 0.05 & 0.19 & $-2.05 * *$ & $-0.81 * *$ \\
\hline BRAGG X LPA 5-3 & 1.62 & 1.58 & -2.40 & -0.18 & 1.14 & 1.45 & -0.02 & 0.02 & -0.41 & -0.81 & $0.49 * *$ & -0.36 & $-1.06 * *$ \\
\hline BRAGG X LPA 13-1 & -1.38 & 1.68 & 1.58 & -0.02 & 0.28 & $4.95 *$ & -0.02 & 0.12 & 1.54 & 0.12 & 0.12 & $1.24 * *$ & 0.16 \\
\hline BRAGG X LPA 18 & -1.78 & -2.32 & 1.56 & $0.54 *$ & $2.52 * *$ & $14.43 * *$ & $0.20 *$ & $-0.83 * *$ & $4.16 * *$ & 1.46 & $-0.33 *$ & $2.62 * *$ & $0.88 * *$ \\
\hline RKS 18 X LPA 5-1 & 0.02 & 0.68 & -0.57 & -0.06 & 1.36 & $10.37 * *$ & -0.10 & $-0.65 *$ & -0.40 & -0.27 & $0.72 * *$ & $1.06 * *$ & $-0.61 *$ \\
\hline RKS 18 X LPA 5-2 & -1.28 & $-3.42 *$ & 2.88 & 0.30 & $3.72 * *$ & $14.55 * *$ & $0.24 * *$ & $1.16 * *$ & $8.33 * *$ & 2.30 & $-0.87 * *$ & $1.23 * *$ & $0.81 * *$ \\
\hline RKS 18 X LPA 5-3 & -0.78 & 0.18 & 0.11 & -0.14 & -0.06 & $-7.47 * *$ & 0.02 & 0.28 & -1.02 & 0.05 & $-1.22 * *$ & -0.18 & 0.47 \\
\hline RKS 18 X LPA 13-1 & 0.72 & 2.28 & 2.09 & -0.38 & -1.42 & $-5.47 *$ & -0.08 & -0.4 & $-2.70 *$ & -1.03 & $1.16 * *$ & $-1.10 * *$ & -0.21 \\
\hline RKS 18 X LPA 18 & 1.32 & 0.28 & $-4.53 *$ & 0.28 & $-3.58 * *$ & $-11.99 * *$ & -0.06 & -0.4 & $-4.21 * *$ & -1.05 & 0.22 & $-1.01 *$ & -0.46 \\
\hline Co 2 X LPA 5-1 & -0.08 & 1.38 & $3.57 *$ & 0.12 & -1.02 & -3.53 & -0.04 & -0.53 & -1.91 & -1.81 & $0.47 * *$ & $1.14 * *$ & 0.36 \\
\hline Co 2 X LPA 5-2 & -0.88 & 1.78 & $-4.78 * *$ & -0.32 & $-3.56 * *$ & $-15.85 * *$ & $-0.20 *$ & $-1.00 * *$ & $-7.51 * *$ & $-3.43 *$ & $0.79 * *$ & $-0.95 *$ & $-1.70 * *$ \\
\hline Co 2 X LPA 5-3 & 0.12 & -0.12 & 2.05 & 0.34 & $2.76 * *$ & $15.73 * *$ & $0.28 * *$ & $0.65 *$ & $7.75 * *$ & $4.37 * *$ & $-0.63 * *$ & $1.15 * *$ & $1.89 * *$ \\
\hline Co 2 X LPA $13-1$ & 0.12 & -2.52 & -0.67 & 0.3 & 0.2 & -1.57 & -0.02 & 0.01 & -0.76 & 1.21 & -0.2 & $-0.94 *$ & $-0.58 *$ \\
\hline Co 2 X LPA 18 & 0.72 & -0.52 & -0.19 & -0.44 & $1.64 *$ & $5.21 *$ & 0 & $0.86 * *$ & $2.44 *$ & -0.34 & $-0.43 * *$ & -0.4 & 0.03 \\
\hline
\end{tabular}

Table.7 Continued...

\begin{tabular}{|c|c|c|c|c|c|c|c|c|c|c|c|c|c|}
\hline Hybrids & $\begin{array}{c}\text { Days to } 50 \\
\text { per cent } \\
\text { flowering }\end{array}$ & $\begin{array}{l}\text { Days to } \\
\text { maturity }\end{array}$ & $\begin{array}{l}\text { Plant } \\
\text { height }\end{array}$ & $\begin{array}{c}\begin{array}{c}\text { Number } \\
\text { of } \\
\text { branches }\end{array} \\
\text { per plant }\end{array}$ & $\begin{array}{c}\text { Number } \\
\text { of } \\
\text { clusters } \\
\text { per } \\
\text { plant }\end{array}$ & $\begin{array}{c}\text { Number } \\
\text { of pods } \\
\text { per } \\
\text { plant }\end{array}$ & $\begin{array}{l}\text { Number } \\
\text { of seeds } \\
\text { per pod }\end{array}$ & $\begin{array}{c}\text { Hundred } \\
\text { seed } \\
\text { weight }\end{array}$ & $\begin{array}{c}\text { Seed } \\
\text { yield } \\
\text { per } \\
\text { plant }\end{array}$ & $\begin{array}{c}\text { Harvest } \\
\text { index }\end{array}$ & $\begin{array}{l}\text { Phytic } \\
\text { acid } \\
\text { content }\end{array}$ & $\begin{array}{l}\text { Protein } \\
\text { content }\end{array}$ & $\begin{array}{c}\text { Oil } \\
\text { content }\end{array}$ \\
\hline Co (Soy) 3 X LPA 5-1 & 0.02 & -0.42 & -1.15 & 0.3 & 0.5 & $9.41 * *$ & $0.20 *$ & -0.22 & $3.63 * *$ & $3.77 *$ & $-0.49 * *$ & $-1.05 * *$ & $0.85 * *$ \\
\hline Co (Soy) 3 X LPA 5-2 & 0.22 & -0.52 & 3.1 & 0.46 & $1.96 *$ & $8.49 * *$ & 0.14 & 0.53 & $4.96 * *$ & 1.2 & -0.07 & 0.68 & $0.90 * *$ \\
\hline Co (Soy) 3 X LPA 5-3 & -0.28 & -0.92 & 0.83 & 0.12 & $-2.42 * *$ & $-8.73 * *$ & $-0.18 *$ & $-0.67 *$ & $-4.80 * *$ & -2.5 & $0.66 * *$ & -0.03 & $-0.73 * *$ \\
\hline Co (Soy) 3 X LPA 13-1 & -0.28 & -0.82 & -2.49 & 0.08 & 1.32 & 1.57 & 0.12 & 0.41 & 1.81 & -0.11 & $-1.03 * *$ & $1.18 * *$ & 0.46 \\
\hline Co (Soy) 3 X LPA 18 & 0.32 & 2.68 & -0.31 & $-0.96 * *$ & -1.34 & $-10.75 * *$ & $-0.26 * *$ & -0.06 & $-5.61 * *$ & -2.36 & $0.93 * *$ & $-0.79 *$ & $-1.48 * *$ \\
\hline JS 335 X LPA 5-1 & -0.88 & 0.78 & $-3.79 *$ & -0.36 & 0.42 & $-7.45 * *$ & -0.1 & -0.34 & $-2.84 *$ & -0.86 & -0.22 & 0.29 & $-1.43 * *$ \\
\hline JS 335 X LPA 5-2 & 1.32 & 0.68 & 1.46 & -0.1 & 0.58 & $4.83 *$ & 0.04 & 0.35 & 1.02 & -0.13 & -0.04 & $1.09 * *$ & $0.81 * *$ \\
\hline JS 335 X LPA 5-3 & -0.68 & -0.72 & -0.61 & -0.14 & -1.4 & -0.99 & -0.08 & -0.28 & -1.52 & -1.11 & $0.70 * *$ & -0.57 & $-0.57 *$ \\
\hline JS 335 X LPA 13-1 & 0.82 & -0.62 & -0.53 & 0.02 & -0.36 & 0.51 & 0.02 & -0.14 & 0.11 & -0.18 & -0.05 & -0.38 & 0.17 \\
\hline JS 335 X LPA 18 & -0.58 & -0.12 & $3.45 *$ & $0.58 *$ & 0.78 & 3.09 & 0.14 & 0.42 & $3.23 * *$ & 2.29 & $-0.38 *$ & -0.42 & $1.02 * *$ \\
\hline S.E. (sca effects) & 1.65 & 1.54 & 1.65 & 0.23 & 0.72 & 2.17 & 0.08 & 0.26 & 1.04 & 1.45 & 0.14 & 0.37 & 0.23 \\
\hline
\end{tabular}


Among the other high yielding crosses, RKS $18 \times$ LPA 5-2 exhibited significant positive sca effects for number of clusters per plant, number of pods per plant, number of seeds per pod, hundred seed weight, seed yield per plant, protein content and oil content coupled with negative $s c a$ effects for days to maturity and phytic acid content. It indicated the presence of non-additive gene action and hence selection can be effective in this cross in $\mathrm{F}_{2}$ or later generations. The cross Co (Soy) $3 \times$ LPA 5-2 recorded significant positive sca effects for number of clusters per plant, number of pods per plant, seed yield per plant and oil content. The cross Co (Soy) $3 \times$ LPA 5-1 recorded significant positive sca effects for number of pods per plant, number of seeds per pod, seed yield per plant, harvest index and oil content coupled with significant negative sca effects for protein and phytic acid content. The cross Co (Soy) $3 \times$ LPA 131 recorded positive sca for protein content and negative sca for phytic acid content and non-significant sca effects for other characters. The significant sca values indicating that above crosses could be best utilized for heterosis breeding due to nonadditive gene action (Table 7). Similar results of desirable sca effects have been reported for seed yield and phytic acid content by Singh et al., (2010) and Ahmad et al., (2013). The crosses RKS $18 \times$ LPA 5-2, Co (Soy) $3 \times$ LPA 5-2 and Co (Soy) $3 \times$ LPA 5-1, Co (Soy) $3 \times$ LPA 13-1 exhibited superior per se performance and one of the parent possessed good general combining ability for yield related traits which indicates additive type of gene action. Hence, selection can be exercised in early generation itself in this cross. This cross could be exploited by pedigree method to obtain segregants with high yield and low phytic acid content.

\section{Acknowledgement}

The authors gratefully acknowledge Board of Research in Nuclear Sciences (BRNS), Mumbai, for the financial assistance provided for this study under the GOI scheme "Development of Low Phytate Soybean [Glycine $\max$ L. (Merr.)] through Induced Mutagenesis".

\section{References}

Ahmad, I., F. Muhammad and M. Aurangzeb. 2013. Breeding bread wheat for low phytic acid using full diallel crosses. Sarhad J. Agric., 29(1): 33-42.

Dhillon, B.S. 1975. The applicability of partial diallel crosses in plant breedingA review. Crop Improv., 2: 1-7.

Kempthorne, O. 1957. An Introduction to Genetic Statistics. John Wiley and Sons Inc., New York, 545.

Mahesh, J., S.R. Ramgiry and S. K. Yadav. 2014. Study of Gene Action and Combining Ability for Fhysiomorphic and Yield Characters in Soybean [Glycine max (L.) Merrill]. Soybean Research (Special Issue Number 2): 5763.

Singh, P.K., C.V. Kumar and B. Bandana. 2010. Effects of salicylic acid on seedling growth and nitrogen metabolism in cucumber (Cucumis sativus L.). J. Stress Physiol. Biochem., 6 (3): 102-113.

\section{How to cite this article:}

Nagarajan, D., T. Kalaimagal and Murugan, E. 2017. Combining Ability Analysis for Yield Component and Biochemical Traits in Soybean [Glycine max (L.) Merrill]. Int.J.Curr.Microbiol.App.Sci. 6(11): 2894-2901. doi: https://doi.org/10.20546/ijcmas.2017.611.341 